\title{
ZUR SPEZIALISIERUNG DES ARBEITSGEDÄCHTNISSES AUF DIE SATZVERARBEITUNGSPROZESSE
}

\section{Einführung}

Arbeitsgedächtnis, Kurzzeitgedächtnis oder Verarbeitungsressourcen werden oft als gleichwertige Konzepte betrachtet. Zwischen den einzelnen Begriffen bestehen jedoch beträchtliche Unterschiede. Der Grund für die so große Diversität der Auffassungen zum temporären Speichersystem liegt darin, dass die Betrachtungsweise des Gedächtnisses mit der Zeit zahlreichen Veränderungen unterlag, wobei diese immer größere Facetten umfassten.

Die von Atkinson und Shiffrin (1968) eingeführte Distinktion zwischen dem Langzeitgedächtnis, in dem Erinnerungen von Fakten und autobiographischen Ereignissen für Jahre abgespeichert werden, und dem Kurzzeitgedächtnis, das dem Behalten der Repräsentationen für kurze Zeitabschnitte dient, hat strukturelle Aspekte des kognitiven Speichersystems in den Vordergrund gerückt. Das Kurzzeitgedächtnis wurde als einfacher Puffer angesehen, in dem temporäre Repräsentationen so lange aufrechterhalten werden, bis sie gelöscht oder durch andere temporäre Repräsentationen ersetzt werden. Die temporären Repräsentationen können auch aus dem Kurzzeitgedächtnis in das Langzeitgedächtnis gelangen. Der Überführungsprozess sollte nach dem oben genannten Modell durch das sog. erhaltende Wiederholen (Rehearsal) erfolgen.

Die ausschließlich strukturelle Betrachtungsweise des Kurzzeitgedächtnisses erwies sich bald als zu einfach und unzureichend. Insbesondere konnte im Rahmen des Modells von Atkinson und Shiffrin (1968) der Einfluss des Langzeitgedächtnisses auf die Kapazität des Arbeitsgedächtnisses und die Dauer der Aufbewahrung von Repräsentationen (Retentionsintervall) nicht erklärt werden. Der Grund dafür ist darin zu sehen, dass das Kurzzeitgedächtnis im Modell von Atkinson und Shiffrin (1968) von dem Langzeitgedächtnis als funktional unabhängig angesetzt

${ }^{*}$ Dr. Jolanta Sękowska, Maria-Curie-Skłodowska-Universität, Humanistische Fakultät, Institut für Germanistik und Angewandte Linguistik, 20-031 Lublin, Pl. Marii Skłodowskiej-Curie 4A. 
wird. Außerdem ist unklar, wie Repräsentationen aus dem Kurzzeitgedächtnis in das Langzeitgedächtnis transferiert werden sollen.

Im Gegensatz zu Atkinson und Shiffrin (1968) werten Baddeley und Hitch (1974, 1994) sowie Baddeley (1986, 1992, 1995, 2003) das Kurzzeitgedächtnis nicht als passiven Speicher, der in die Wissensverarbeitungsprozesse involviert ist. Stattdessen wird ein Arbeitsgedächtnis vorgeschlagen, das nicht nur für die Ablage, sondern auch für die Verarbeitung der Repräsentationen zuständig ist:

Working memory may be defined as the system for the temporary maintenance and manipulation of information, necessary for the performance of such complex cognitive activities as comprehension, learning and reasoning (Baddeley 1992, S. 281).

Baddeleys Konzept ist das erste modulare Modell der funktionellen Architektur des menschlichen Gedächtnisses. Die ursprünglich definierten Module waren die phonologische Schleife (phonological loop), der visuell-räumliche Notizblock (visuospatial sketchpad) und die zentrale Exekutive. Bei der Sprachverarbeitung wird der sprachliche Input in einen phonologischen Code übersetzt und in der phonologischen Schleife abgelegt. Die phonologischen Repräsentationen werden in der phonologischen Schleife durch Rehearsal bereitgehalten. Falls dieser Prozess unterdrückt wird, zerfallen die Gedächtnisspuren in der phonologischen Schleife innerhalb 1-2 Sekunden, sodass sie nicht mehr verwendet werden können. Demgegenüber dient der visuell-räumliche Notizblock der temporären Speicherung von visuellen Wahrnehmungen. Die beiden Subsysteme des Arbeitsgedächtnisses werden von der modalitätsunspezifischen Überwachungsinstanz, der sog. zentralen Exekutive, kontrolliert, die eine Art Aufmerksamkeitssystem bildet, indem sie die Verarbeitungsprioritäten vorgibt, die routinisierten Verarbeitungsprozesse unterbricht und die nichtroutinisierten überwacht.

Das Arbeitsgedächtnis übernimmt nach Baddeley sowohl Speicherungs- als auch Verarbeitungsfunktionen, wodurch temporäres Beibehalten der aktivierten Repräsentationen im Gedächtnis und ihre Manipulierung ermöglicht wird. Die aktivierten Repräsentationen können auch dem Bewusstsein zugänglich sein (Baddeley 1986). Somit liegen dem Arbeitsgedächtnis mehrere kognitive Funktionen zugrunde, einschließlich des Denkens, Problemlösens sowie der meisten Aspekte der Sprachverarbeitung. Die Anforderungen an das Arbeitsgedächtnis variieren je nach Situationen und Individuen, wobei sich letztere in der Sachkenntnis oder in den kognitiven Fähigkeiten unterscheiden. Obwohl es einen beträchtlichen Konsens dahingehend gibt, dass das Arbeitsgedächtnis eine zentrale Rolle bei der kognitiven Verarbeitung spielt, herrscht Unstimmigkeit hinsichtlich der Frage, wie es organisiert und inwieweit diese Organisation domänenspezifisch ist. Insbesondere geht es hier um die Spezialisierung des Arbeitsgedächtnisses auf die Satzverarbeitungsprozesse, deren Kontinuität bei der Verarbeitung von komplexen Inputdaten durch das Arbeitsgedächtnis sichergestellt werden muss. 


\section{Ist das Arbeitsgedächtnis ein vom Langzeitgedächtnis separates System?}

Kontrovers ist, ob das Arbeitsgedächtnis als eine distinkte, gerade aktivierte strukturelle Komponente des Langzeitgedächtnisses aufgefasst werden soll. Die einschlägige Fachliteratur liefert drei unterschiedliche Antworten auf diese Frage. Laut Baddeley und Hitch (1974) ist das Arbeitsgedächtnis ein komplexes System, dessen Bestandteile strukturell unabhängig voneinander funktionieren und vom Langzeitgedächtnis getrennt sind. Dieser Ansicht zufolge ist das Arbeitsgedächtnis ein „Ort“, wo ankommende Inputdaten strukturiert werden. Der lexikalische Abruf erfolgt, indem die langfristige Kodierung einer lexikalischen Einheit im Arbeitsgedächtnis kopiert oder gebunden wird.

Ein wichtiger Punkt innerhalb der Kritik an Baddeleys Modell bezieht sich darauf, dass es das Phänomen des Chunkings, d.h. des Zusammenfügens einzelner Einheiten zu größeren sinnhaften Einheiten bei gleichzeitiger Abgrenzung von anderen Einheiten, nicht erklären kann. Das Chunking lässt das Vorhandensein einer Verbindung zwischen dem Arbeits- und Langzeitgedächtnis vermuten. Deswegen wird der zweiten Auffassung zufolge der Inhalt des Arbeitsgedächtnisses durch die Aktivierung der Repräsentationen im Langzeitgedächtnis determiniert (Logie 1995). Diese Position wiederum knüpft an die Konzeption von Anderson (1972) an und wird u.a. auch von Stolzfus, Hasher und Zacks (1996) vertreten: Der kurzfristige Speicher (oder das Arbeitsgedächtnis) wäre dann ein distinkter struktureller Bestandteil des Langzeitgedächtnisses und sein Inhalt würde durch einen Satz von Knoten bestimmt, die aktuell in der interpretativen Verarbeitungsphase im Langzeitgedächtnis aktiviert werden. Interessanterweise wird hier nicht beachtet, dass die Verbindung von einfachen lexikalischen Einheiten zu größeren sinnhaften Einheiten bei der Satzverarbeitung auf einem komplexeren Mechanismus als dem des Chunkings beruhen kann, worauf im Verlauf des vorliegenden Beitrags noch eingegangen wird.

Nach der dritten, radikaleren Auffassung ist das Arbeitsgedächtnis ein Subsystem des Langzeitgedächtnisses und keine strukturell distinkte Komponente. Diese Konzeption des Arbeitsgedächtnisses wurde ursprünglich von Anderson \& Bower (1973) verfochten und darüber hinaus und auch für die konnektionistische Architektur der Sprachverarbeitung als geltend postuliert (z.B. MacDonald et al. 1994; MacDonald, Christiansen 2002).

Die Funktion des Arbeitsgedächtnisses im Embedded-Processes-Modell von Cowan (1997, 2001; Cowan et al. 2002, 2003) besteht darin, die Repräsentationen im Langzeitgedächtnis in einem hochaktivierten und dadurch gut zugänglichen Zustand zu halten. Cowan betrachtet das Arbeitsgedächtnis somit als eine Menge der aktivierten Repräsentationen. Zu beachten ist dabei aber, dass sich auch schon eine Untermenge der aktivierten Repräsentationen zu einem gegebenen Zeitpunkt 
im Fokus der Aufmerksamkeit befinden kann und nicht alle Gedächtnisprozesse den fokussierten Input betreffen. Implizite Gedächtnisprozesse können auch ohne Aufmerksamkeit initiiert werden.

In Bezug auf die Kapazität des Arbeitsgedächtnisses und sein Retentionsintervall sehen Cowan wie Baddeley eine Beschränkung vor. Während jedoch Baddeley für das Rehearsal 2 Sekunden Latenz nimmt, hängt für Cowan die Kapazität des Arbeitsgedächtnisses grundsätzlich von der Anzahl der abgespeicherten Elemente ab. Nach Cowan (2001) liegt diese Grenze bei 4 Elementen. Ähnlich wie im modularen Modell von Baddeley verblassen die Repräsentationen nach 20 Sekunden, wenn sie nicht reaktiviert werden.

\section{Argumente gegen den unitären Ansatz für Satzverstehensprozesse}

Die Satzstruktur kann nicht als Ganzes aus dem Arbeitsgedächtnis abgerufen werden, sondern wird aus kleineren Elementen aufgebaut. Beim Satzverstehen erfordert dies, dass die Lemmata und syntaktischen Produkte des partiellen Strukturaufbaus im Arbeitsgedächtnis so lange aufbewahrt werden, bis sie syntaktische Slots besetzen. Um diesen Prozess zu bewerkstelligen, ist ein temporäres Speichersystem notwendig. Zwischen den syntaktischen Strukturaufbauoperationen (Parsingprozessen) und dem Arbeitsgedächtnis scheint eine Relation zu bestehen. Den dargestellten Arbeitsgedächtnismodellen fehlt es aber an der Präzision, die notwendig wäre, um die Parsing-Arbeitsgedächtnis-Relation zu erklären.

Problematisch für den unitären Ansatz ist insbesondere die Reduktion des sprachspezifischen auf das generelle Arbeitsgedächtnis aus falsch verstandenen Ökonomiegründen. Das in die Satzverarbeitungsprozesse involvierte Arbeitsgedächtnis operiert nicht innerhalb von Sekunden, sondern innerhalb von Zehntelsekunden. Anders als das generelle Arbeitsgedächtnis oder die Aufmerksamkeitssysteme dient das sprachspezifische Arbeitsgedächtnis automatischen Prozessen, die zu schnell ablaufen, als dass sie der Introspektion oder der bewussten Aufmerksamkeit zugänglich wären. Dies ist ein überzeugendes Argument zugunsten der modularen Auffassung.

Die Verbindung der Domänenunspezifität des Arbeitsgedächtnisses mit der konnektionistischen Architektur der Satzverarbeitung ist ebenfalls umstritten. Beispielsweise wendet Jackendoff (2007) dagegen ein, dass dadurch der syntaktische Strukturaufbau verhindert würde, weil die im Laufe der Spracheverarbeitung sequenziell wahrgenommenen lexikalischen Einheiten zwar aktiviert werden, doch gewissermaßen in der Luft hängen, da es ohne Annahme eines syntaktischen Moduls keine Möglichkeit gibt, sie syntaktisch zu verbinden: the dog chased a cat, the cat chased a dog und dog cat a chased aktivieren die gleichen lexikalischen Einheiten. Jackendoff (2007) verweist auch darauf, dass die Verarbeitung von Sätzen wie my cat likes your cat, in denen die gleiche lexikalische Einheit 
zweimal erscheint, im Rahmen des Konnektionismus nur schwer nachzuvollziehen ist. Für cat gibt es nämlich in der konnektionistichen Architektur (vermutlich) nur einen Knoten, dennoch bezieht sich der Satz auf zwei distinkte Katzen. Wird das Arbeitsgedächtnis vom Langzeitgedächtnis losgelöst, dann bleiben die genannten Probleme aus. Für das gleiche lexikalische Material liegen im Arbeitsgedächtnis zwei Kopien vor, von denen jede ein eigenes Verhältnis zum Sprachmaterial unterhält.

Im Zusammenhang mit dem unitären Ansatz entsteht nach Jackendoff (2007) weitere Schwierigkeit, und zwar die Unterscheidung zwischen der temporären und dauerhaften lexikalischen Bindung. Die Satzstruktur wird im konnektionistischen Modell der Satzverarbeitung von MacDonald et al. (1994) durch eine Bindung der lexikalischen Einträge aufgebaut. Die Phrasen weichen jedoch in ihrem Bindungspotential voneinander ab. Im Falle der Phrase throw the shovel verläuft die Bindung zwischen dem Verb und dem direkten Objekt vorübergehend, wogegen sie in der Phrase kick the bucket als fester Bestandteil zum Teil des lexikalischen Wissens gehört und folglich als dauerhaft gekennzeichnet ist. Diese Differenzierung wurde von MacDonald et al. (1994) nicht berücksichtigt, was umso bedauerlicher ist, wenn man bedenkt, dass sich das Problem durch die Annahme der Existenz eines separaten Arbeitsgedächtnissystems leicht lösen ließe. In beiden genannten Beispielen erfolgt eine Bindung im Arbeitsgedächtnis, aber nur im Falle von kick the bucket schlägt diese Bindung aufs Langzeitgedächtnis durch.

\section{Gibt es ein sprachspezifisches Arbeitsgedächtnis?}

Die Diskussion um die Domänenspezifität des Arbeitsgedächtnisses wirft die grundlegende Frage nach seiner Modularität auf: Besteht das Arbeitsgedächtnis aus Subsystemen, die unterschiedlichen Aufgabentypen gewidmet sind? Ist das Subsystem des Arbeitsgedächtnisses, das in die Verarbeitung syntaktischen Wissens einbezogen wird, auch in die bewusste Wissensverarbeitung involviert? Würde man davon ausgehen, dass es ein sprachunspezifisches Arbeitsgedächtnis gäbe, das an das Aufmerksamkeitssystem gekoppelt ist, so hätte dies zur Folge, dass die individuelle Variation im Arbeitsgedächtnis der potentiellen in der Satzverarbeitung zugrunde liegt. Die Frage betrifft somit das Maß, in dem die individuelle Variation in der domänenunspezifischen Fähigkeit zum temporären Behalten der Wissensrepräsentationen zentral für das Satzverstehen ist.

Laut Just und Carpenter (1992; vgl. auch Carpenter, Miyake, Just 1994) ist das Arbeitsgedächtnis nicht auf die Syntaxverarbeitung spezialisiert (capacity theory of comprehension). Das deklarative Wissen wie das Lexikon und das prozedurale Wissen als Condition-Action-Regeln werden im Langzeitgedächtnis gespeichert. Ein separater Arbeitsgedächtnisraum dient der Verarbeitung des eingehenden Inputs und der temporären Speicherung der durch fortlaufende Komputation zur 
Verfügung gestellten Produkte. Die Speicherungs- und Verarbeitungsfunktionen, die für die Satzverarbeitung notwendig sind, werden hier durch Aktivierung erfüllt, während die Repräsentationen ohne genügende Aktivierung gelöscht werden.

Just und Carpenter (1992) führen als Argument gegen die Modularität ins Feld, dass das Parsing nicht als ein syntaktisches Modul im Sinne Fodors (1983) zu gelten hat ${ }^{1}$.Wenn gezeigt werden kann, dass die Kapazität des Arbeitsgedächtnisses die Parsingprozesse beeinflusst, so lässt sich nach Just und Carpenter (1992) auf das Konzept der Modularität verzichten, um Parsing zu erklären. Ähnlich sollen die Befunde aus den Studien, die einen Einfluss der Arbeitsgedächtniskapazität auf die syntaktische Verarbeitung nachgewiesen haben, das Argument erhärten, wonach syntaktische Verarbeitungsprozesse kein Modul der Satzverarbeitung bilden. Das, was ein Effekt der Modularität in der Satzverarbeitung zu sein scheint, ist nach Just und Carpenter (1992) ein Nebenprodukt des Kapazitätseffekts. Die Differenzen im generellen Arbeitsgedächtnis induzieren bei Hörern/Lesern einer Sprache unterschiedliche Strategien der Online-Satzverarbeitung. Demnach gebe es also keinen universellen Mechanismus, dem die Parsingprozesse unterliegen. Die grundlegende Frage nach der Architektur der Sprachverarbeitung wird somit auf die Effekte der kognitiven Kapazität zurückgeführt. Dies ist eine wichtige Annahme, die einer genaueren Untersuchung bedarf, weil sie weitreichende Folgen für die Modelle der Satzverarbeitung und des Agrammatismus im Allgemeinen zeitigt.

Besondere Beachtung ist der Tatsache zu schenken, dass sich die Theorie von Just und Carpenter (1992) auf die aus Messungen der Lesespanne gewonnenen Erkenntnisse stützt (reading span task von Daneman, Carpenter 1980). Die Wissenschaftler präsentierten den Probanden reduzierte Relativsätze wie The defendant examined by the lawyer shocked the jury. Wenn die initiale NP ein plausibler Agens des Hauptverbs war (The defendant examined...), zeigten die Probanden sowohl mit einer niedrigeren als auch mit einer größeren Lesespanne verzögerte Lesezeiten bei dem disambiguierenden Element by. Die Leistung der beiden Probandengruppen variierte aber, sobald die initiale NP einen implausiblen Agens beinhaltete (The evidence examined...). Infolge der Manipulation der semantischen Plausibilität wurden die Lesezeiten bei Probanden mit einer größeren Lesepanne reduziert, wogegen sie bei Probanden mit einer niedrigeren Lesespanne konstant gehalten wurden. Laut Just und Carpenter (1992) bringen die Probanden mit einer größeren Lesepanne im Gegensatz zu den Probanden mit einer niedrigeren Lesespanne die Arbeitsgedächtniskapazität mit, die notwendig ist, um das Plausibilitätswissen im Laufe des Satzverstehens zu nutzen².

Die Interpretation der Befunde, auf deren Grundlage Just und Carpenter (1992) ihre Theorie des Satzverstehens konzipiert haben, kann sich aber als falsch

${ }^{1}$ Gemäß der modularistischen Perspektive können zu gegebener Zeit nur einige Wissenstypen dem Satzverarbeitungsmechanismus unterworfen werden.

${ }^{2}$ Andere Studien berichten über ähnliche Resultate (z.B. Pearlmutter \& MacDonald 1995). 
herausstellen. Die Tatsache, dass die Probanden mit einer größeren Lesespanne semantische Erwartungen zeigen, kann lediglich als Indiz dafür gedeutet werden, dass sie sehr schnell semantischen Hinweisen zur Satzinterpretation folgen. Demgegenüber können sich die Probanden mit einer niedrigeren Lesespanne in erster Linie Zugang zu syntaktischen Prinzipien verschaffen, aber dafür wegen der reduzierten Arbeitsgedächtniskapazität nicht so schnell auf das semantische Wissen zugreifen. Vielleicht erfordert syntaktisches Wissen eine geringere Belastung des Arbeitsgedächtnisses oder wird schneller aktiviert als semantisches. Die bevorzugte Nutzung des einen Wissenstyps könnte dann als Indiz für die Modularität des Satzverarbeitungssystems gewertet werden.

Die Ansichten hinsichtlich des Bestehens einer Korrelation zwischen der Arbeitsgedächtnisspanne und der syntaktischen Verarbeitung haben sich dank Caplan \& Waters (1999, 2002, vgl. auch Waters \& Caplan 1996a, b, 2004; Caplan, Waters \& DeDe 2007) grundsätzlich gewandelt. In ihren Untersuchungen wiederholten sie die Experimente, stießen dabei aber auf keine signifikante Relation zwischen der Arbeitsgedächtnisspanne und der Leistung beim Satzverstehen. Demgegenüber fordern Caplan \& Waters $(1999,2002)$ mit ihrer SSIR-Theorie (separate-sentence-interpretation-resource theory) eine Gliederung des Arbeitsgedächtnisses in ein spezialisiertes verbales Arbeitsgedächtnis, das die erste Phase der Satzverarbeitung, die sog. interpretative Verarbeitung, unterstützt, und ein generelles verbales Arbeitsgedächtnis, das in die zweite Phase der Satzverarbeitung, die sog. post-interpretative Verarbeitung, eingreift ${ }^{3}$. Die interpretative Verarbeitung soll sich auf die Extraktion der Bedeutung aus dem sprachlichen Input beziehen sowie auf die Worterkennung und die Auswertung von Wortbedeutung und syntaktischen Eigenschaften, auf das Konstruieren von prosodischen und syntaktischen Repräsentationen und das Zuweisen von thematischen Rollen, des Fokus und anderer semantischer Aspekte. Die post-interpretative Verarbeitung soll dagegen bewusste kontrollierte Prozesse umfassen wie den Gebrauch des Weltwissens in der Textinterpretation, die Erinnerungen an den Satzinhalt sowie die Planung von Aktivitäten, die auf der Bedeutung von Sätzen und Texten basieren. Dieses Subsystem des Arbeitsgedächtnisses kann sich einer individuellen Variation ausgesetzt sehen. Die Domänenspezifität des Arbeitsgedächtnisses wird hier somit als eine kognitive Spezialisierung auf die interpretative Satzverarbeitung verstanden. Zu beachten ist aber, dass der Vorschlag von Caplan und Waters (1999, 2002) zwar eine Spezialisierung des Arbeitsgedächtnisses auf das Satzverstehen im Allgemeinen, nicht jedoch auf Parsingprozesse im Besonderen vorsieht. Zwar nehmen die Forscher zwei separate Subsysteme des Arbeitsgedächtnisses an, aber

${ }^{3}$ Unterstützung erfahren sie von der neurophysiologischen Forschung. Die Patienten mit einer deutlich reduzierten Arbeitsgedächtniskapazität behalten oft die Fähigkeit, syntaktisch komplexe Strukturen zu verstehen und zu produzieren (z.B. Butterworth et al. 1986; Waters, Caplan, Hildebrandt 1991; Martin 1993). 
sie schließen eine weitere Unterteilung auf der Grundlage der empirischen Untersuchungen nicht aus ${ }^{4}$.

Darüber hinaus verweisen Caplan und Waters $(1999,2002)$ auf eine Reihe von Problemen sowohl in Bezug auf das experimentelle Design zur Untersuchung der Arbeitsgedächtniskapazität als auch auf die Konzeptualisierung des Arbeitsgedächtnisses für Satzverstehensprozesse. Insbesondere betonen sie, dass mit den Messungen der Lesepanne eine bewusste Koordination von komplexen Fähigkeiten wie etwa das Aufbewahren des letzten Wortes beim Lesen des nächsten Satzes untersucht wird. Die Syntaxverarbeitung gründet jedoch in unbewussten automatischen Prozessen, was zugleich nicht ausschließt, dass sich die individuellen Unterschiede in dem generellen domänenunspezifischen Arbeitsgedächtnis auf die Ergebnisse der Offline-Satzverarbeitung auswirken.

\section{Das Arbeitsgedächtnis und die syntaktische Komplexität im Lichte der neurophysiologischen Studien und bildgebenden Verfahren}

Das Satzverstehen hängt entscheidend von der Rekonstruktion der grammatischen Relationen zwischen dem Prädikat und seinen Argumenten ab, indem den Inputwörtern eine hierarchische Struktur zugewiesen wird (z.B. Frazier 1987, 1988, 1990). Aus der psycho- und neurolinguistischen Forschung erreichen uns eindeutige Signale, die darauf hindeuten, dass die Verarbeitungsschwierigkeiten je nach syntaktischer Komplexität variieren, und dies sogar unabhängig vom Satztyp (beim Scrambling: Friederici, Mecklinger 1996; Meng et al. 1999; bei der Voranstellung des Pronomens im Mittelfeld: Bader, Meng 1999; bei wh-Fragen: Schlesewsky et al. 1999; bei Topikalisierungen: Frisch et al. 2002 und in Relativsätzen: Schreifers et al. 1995; Lin, Bever 2006). Ein prototypisches Beispiel für Sätze, die sich in der syntaktischen Komplexität voneinander unterscheiden und deren Verarbeitung gleichzeitig mit unterschiedlichem Verarbeitungsaufwand korrespondiert, sind Sätze mit Subjekt-vor-Objekt- und Objekt-vor-SubjektAbfolge. Die syntaktische Verarbeitung (Parsing) verläuft relativ problemlos, wenn die Inputwörter in der kanonischen Subjekt-vor-Objekt-Abfolge erscheinen. Demgegenüber wirken sich die Wortstellungvariationen negativ auf die Leistung der Probanden beim Satzverstehen aus.

Eine Operation, die eine Bildung der nicht-kanonischen Wortfolgen durch Bewegung eines Arguments in die satzinitiale Position zulässt, wird in der generativen

${ }^{4}$ So vermutet Carruthers (2006) eine immense Anzahl von Arbeitsgedächtnissystemen, die mit Verarbeitungsmodulen gekoppelt sind. Diesen Schluss zieht Carruthers (2006) daraus, dass die Berücksichtigung der Kapazität für komputationelle Prozesse immer eine Inputrepräsentation voraussetzt sowie die Fähigkeit, einige Zwischenrepräsentationen für kurze Zeit zu behalten, bevor die nächste Komputationsphase beginnt. 
Grammatik als $w h$-Bewegung bezeichnet (z.B. Chomsky 1981). Eine Konstituente, die in die initiale Satzposition verschoben wird, hinterlässt eine phonologisch leere Kategorie (Spur), wobei das extrahierte Element mit der Spur (in der Satzverarbeitungsforschung als gap bezeichnet) koindiziert ist. Die Koindizierung ermöglicht, dass der Hörer oder Leser das dislozierte Element als filler (Füller) für a gap (eine Lücke) identifiziert.

Weniger klar ist, wie syntaktische Merkmale des Füllers nach seiner Identifizierung verarbeitet werden. Die einschlägige Fachliteratur liefert dafür zwei potentielle Erklärungen: Entweder werden die syntaktischen Eigenschaften des Füllers identifiziert und dann in der Spurenposition reaktiviert (Nicol, Swinney 1989; Nicol et al. 1994) oder nach der Identifizierung bleiben sie im Arbeitsgedächtnis aktiviert, bis die Spur erreicht wird (z.B., Clifton, Frazier 1989; Frazier, Flores D’Arcais 1989; Kluender, Kutas 1993; Fanselow, Kliegl, Schlesewsky 1999). Die beiden genannten Vorschläge bringen unterschiedliche Implikationen in Bezug auf die Rolle des Arbeitsgedächtnisses bei der Verarbeitung der syntaktisch komplexen Strukturen mit sich.

In der einschlägigen Fachliteratur herrscht Übereinstimmung darüber, dass das Arbeitsgedächtnis - wegen der sequenziellen Natur des sprachlichen Inputs eine kritische Rolle bei der Rekonstruktion der filler-gap-Abhängigkeiten innerhalb der syntaktischen Satzanalyse spielt. Jedoch gehen die Meinungen auseinander in der Frage der potentiellen Trennung der Arbeitsgedächtnisressourcen, die für die Erstellung der satzinternen Abhängigkeitsrelationen unerlässlich sind, von denen, die der Integration des eintreffenden Inputs in die vorliegende Satzstruktur zugrunde liegen (generelles verbales Arbeitsgedächtnis).

Fiebach, Schlesewsky und Friederici $(2001,2002)$ untersuchten mit der EKPMethode die Verarbeitung der wohlgeformten und unambigen kasusmarkierten deutschen Subjekt- und Objekt-wh-Fragen, in denen der Abstand zwischen dem wh-Füller und seiner gap entweder kurz oder lang war. Die gesammelten Daten wurden mit der Verarbeitung der ob-Fragen untersucht, die keine filler-gap-Abhängigkeiten beinhalten. Die Experimente ergaben, dass das Behalten des dislozierten $w h$-Füllers im Arbeitsgedächtnis eine anhaltende links anteriore Negativität evoziert. Die Befreiung des Arbeitsgedächtnisses von dieser Last und die Integration der dislozierten Konstituente in die Repräsentation der Phrasenstruktur wurden im EKP-Bild durch eine lokale Positivierung an der zweiten Nominalphrase registriert. Interessanterweise waren die beiden EKP-Komponenten voneinander unabhängig, worauf ihre distinkten Zeitdimensionen, Topographien und Polaritäten verweisen. Außerdem wurden sie durch die Länge der wh-Bewegung sowie durch individuelle Unterschiede in der Arbeitsgedächtniskapazität verschieden beeinflusst. Die experimentellen Daten sprechen somit für die Trennung der syntaktischen Gedächtnisprozesse von den Integrationskosten und stehen in Einklang mit dem Postulat, dass der Füller im Arbeitsgedächtnis temporär beibehalten wird (z.B. Frazier \& Flores D’Arcais 1989). 
Die oben genannten Befunde verweisen darauf, dass der Aufbau der fillergap-Relationen syntaktischer Natur ist, d.h., dass der Füller so lange aktiv behalten wird, bis die Lücke lizenziert und die syntaktische Kette zwischen dem Füller und der Lücke hergestellt wird. Der Füller oder mindestens seine syntaktischen Merkmale sind dem Parser zugänglich, wenn der neue Input ununterbrochen verarbeitet wird. Fiebach, Schlesewsky und Friederici $(2001,2002)$ postulieren damit eine Spezialisierung des Arbeitsgedächtnisses auf die Verarbeitung syntaktischer Merkmale (syntaktisches Arbeitsgedächtnis). Sie erheben zugleich den Anspruch, dass die Ablage des Füllers im syntaktischen Arbeitsgedächtnis nicht auf Situationen beschränkt ist, in denen die temporäre Ambiguität des Füllers aufgelöst werden muss, sondern dass der Parser die allgemeine Eigenschaft besitzt, die von den kanonischen strukturellen Position dislozierten Argumente nicht sofort, an der Stelle, wo sie wahrgenommen werden, zu verarbeiten. In solchen Fällen wird die Integration der Argumente in die mentale Repräsentation der Phrasenstruktur so lange verzögert, bis eine Konstituente erscheint, die die Antizipation der Spurenposition ermöglicht (z.B. eine Subjekt-NP).

Die Ergebnisse der Studien von Fiebach, Schlesewsky und Friederici (2001, 2002) widersprechen nicht unbedingt dem Nachweis einer Reaktivierung des Füllers an der gap-Position, der durch Priming-Studien erbracht wird (Nicol, Swinney 1989). Es ist durchaus denkbar, dass nur die syntaktischen Merkmale des Füllers im Arbeitsgedächtnis abgelegt werden. Der Mechanismus würde dann dem Parser ermöglichen, den Füller schnell der potentiellen gap-Position zuzuweisen, anstatt alle Merkmale des Füllers an dieser Position zu analysieren. Dennoch müssen die semantischen Merkmale des Füllers reaktiviert werden, wenn die Lücke identifiziert wird. Die Reaktivierung des Antezedens würde dann in einer späteren Phase der Satzverarbeitung als die Erstellung der syntaktischen Kette zwischen dem Füller und seiner Lücke stattfinden.

\section{Broca-Areal und Arbeitsgedächtnis}

Die ersten bildgebenden Studien zum Satzverstehen assoziierten das Broca-Areal (pars opercularis im linken inferior frontalen Gyrus/BrodmannAreal BA44 und den posterioren Teil von pars triangularis/BA 45) mit der Syntaxverarbeitung. Die Annahme basiert ursprünglich auf den aus den Untersuchungen zur Satzverarbeitung bei Broca-Aphasikern gewonnenen Befunden (Zurif et al. 1972; Caramazza, Zurif 1976). Indies erlauben neuere Forschungen genauere Unterteilungen: Der linke pars opercularis (insbesondere BA44i ${ }^{5}$ ) ist in die Verarbeitung der hierarchischen syntaktischen Strukturen, der pars triangularis (BA45) zusammen mit

${ }^{5}$ Der inferiore Teil des Brodmann-Areals BA44 (Fiebach, Vos, Friederici 2004; Friederici, Fiebach, Schlesewsky et al. 2006). 
dem pars orbitalis (BA 47) hingegen in die Verarbeitung semantischen Wissens einbezogen (z. B. Friederici 2002; Friederici, Kotz 2003). Die genaue Natur der Prozesse, die in dem linken pars opercularis ablaufen, ist jedoch bis heute nicht geklärt. Gemäß der funktionellen Neuroanatomie ist nicht nur die Syntaxverarbeitung (Hashimoto \& Sakai 2002; Ben-Shachar et al. 2003; Friederici, Bahlmann et al. 2006; Bahlmann, Schubotz \& Friederici 2008), sondern auch das nicht-syntaktische Arbeitsgedächtnis (, Jonides 1998) in dem linken präfrontalen Kortex zu lokalisieren. Unklar ist dabei, ob der pars opercularis durch die strukturelle Komplexität an sich oder durch die erhöhte Gedächtnislast bei der Verarbeitung komplexer hierarchischer Strukturen aktiviert wird.

Die neusten Untersuchungen werfen jedoch ein neues Licht auf die komplizierte Relation zwischen der syntaktischen Verarbeitung und den Arbeitsgedächtnisressourcen. Um den Effekt der strukturellen Komplexität von den Arbeitsgedächtniseffekten zu trennen, führten Makuuchi et al. (2009) eine fMRI-Studie zum Satzverstehen im Deutschen durch. Das experimentelle Material erfasste sowohl strukturell komplexe Sätze (mit und ohne hierarchische Struktur) als auch die Variation der Arbeitsgedächtnisbelastung (langer/kurzer Abstand zwischen den syntaktisch abhängigen Elementen, d.h. zwischen dem Subjekt und dem Verb). Die Studie konnte zeigen, dass die Prozesse der Verarbeitung syntaktisch komplexer hierarchischer Strukturen und die Arbeitsgedächtnisbelastung zwar separat angeordnet sind, aber im linken inferior-frontalen Gyrus zusammen operieren. Die Aktivierung im linken pars opercularis variiert infolge der strukturellen Komplexität, während die Aktivierung im linken inferior-frontalen Sulcus durch den Abstand zwischen den relevanten syntaktischen Informationen moduliert wird. Darüber hinaus zeigt die Diffusions-Tensor-Bildgebung, dass die beiden Hirnareale durch Fasern der weißen Substanz verbunden sind (Friederici 2009). Die Ergebnisse verweisen einerseits auf die Trennung der syntaktischen Verarbeitungsaspekte im linken pars opercularis von denen, die der Belastung des Arbeitsgedächtnisses zugrunde liegen und durch Aktivierung im linken inferior-frontalen Sulcus reflektiert werden, andererseits deuten sie ihre neurofunktionelle und-strukturelle Zusammenschaltung an.

\section{Fazit}

Die angeführten Befunde aus den neurophysiologischen und bildgebenden Experimenten sprechen eindeutig für die Existenz eines Subsystems des Arbeitsgedächtnisses, in dem nicht-integrierte syntaktische Merkmale temporär während der Satzanalyse abgelegt werden. Das syntaktische Arbeitsgedächtnis ist in den Aufbau der filler-gap- Abhängigkeiten entscheidend einbezogen und scheint als ein distinktes Modul innerhalb der kognitiven Verarbeitungsressourcen zu gelten, die in die interpretative Satzverarbeitung involviert sind. Die experimentellen 
Daten verweisen darauf, dass das syntaktische Arbeitsgedächtnis, das den komputationellen Kern der Satzverarbeitung ausmacht, im linken pars opercularis und das nicht-syntaktische im linken inferior-frontalen Sulcus anzusiedeln ist. Trotz der neurofunktionellen Trennung des syntaktischen und nicht-syntaktischen Arbeitsgedächtnisses verdient die neuroanatomische Verbindung der beiden Hirnregionen besondere Beachtung.

\section{LITERATURVERZEICHNIS}

Anderson J.R. (1972), FRAN: A simulation model of free recall. In: Bower G.H. (ed.), The psychology of learning and motivation: Advances in research and theory, New York, S. 315-378.

Anderson J.R., Bower, G.H. (1973), Human associative memory, Washington DC.

Atkinson R.G., Shiffrin, R.M. (1968), Human memory: A proposed system and its control processes. In: Spence K.W., Spence J.T. (eds.), The psychology of learning and motivation: Advances in research and theory, New York, S. 89-195.

Baddely A.D. (2003), Working memory and language: an overview. In: Journal of Communication Disorders, Bd. 36, S. 189-208.

Baddeley A.D. (1995), Working memory. In: M.S. Gazzaniga (ed.), The cognitive neurosciences. Campidge, Mass., S. 755-764.

Baddeley, A.D. (1992), Working memory: The interface between memory and cognition. In: Journal of Cognitive Neuroscience, Bd. 4 (3), S. 281-288.

Baddeley A.D. (1986), Working memory, New York.

Baddeley A.D., Hitch, G.J. (1994), Developments in the concept of working memory. In: Neuropsychology, Bd. 8, S. 485-493.

Baddeley A.D., Hitch G.J. (1974), The psychology of learning and motivation, New York.

Bader M., Meng M. (1999), Subject-object ambiguities in German embedded clauses : an acrossthe-board comparison. In: Journal of Psycholinguistic Research, Bd. 28(2), S. 121-143.

Bahlmann J., Schubotz R.I., Mueller J.L., Koester D., Friederici A.D. (2009), Neural circuits of hierarchical visuo-spatial sequence processing. In: Brain Research, Bd. 1298, S. 161-70.

Ben-Shachar M., Hendler T., Kahn I., Ben-Bashat D., Grodzinsky Y. (2003), The neural reality of syntactic transformations: Evidence from functional magnetic resonance imaging. In: Psychological Science, Bd. 14, S. 433-440.

Butterworth B., Campbell R., Howard D. (1986), The uses of short-term memory: A case study. In: Quarterly Journal of Experimental Psychology, Bd. 38A, S. 705-737.

Caplan D., Waters G. (1999), Verbal working memory and sentence comprehension, Behavioral and Brain Sciences, Bd. 22, S. 77-94.

Caplan D., Waters G. (2002), Working memory and connectionist models of parsing: A response to MacDonald and Christiansen. In: Psychological Review, Bd. 109, S. 66-74.

Caplan D., Waters G., DeDe G. (2007), Specialized Verbal Working Memory for Language Comprehension. In: Conway A.R.A., Jarrold C., Kane M.J., Miyake A., Towse J.N., (eds.) (2007), Variation in Working Memory, Oxford, S. 272-302.

Caramazza A., Zurif E.B (1976), Dissociation of algorithmic and heuristic processes in language comprehension. In: Brain and Language, Bd. 3, S. 572-582.

Carpenter P.A., Miyake A., Just M.A. (1994), Working memory constraints in comprehension: Evidence from individual differences, aphasia, and aging. In: M.A. Gernsbacher (ed.), Handbook of psycholinguistics, San Diego CA, S. 1075-1122.

Carruthers P. (2006), The architecture of the mind. Massive modularity and the flexibility of thought, Oxford. 
Chomsky N (1981), Lectures on government and binding. The Pisa lectures, Dordrecht.

Clifton Ch., Frazier L. (1989), Comprehending sentences with long-distance dependencies, In: Carlson G.N., Tanenhaus M.K. (eds.), Linguistic structure in language processing, Dordrecht, S. $273-317$.

Cowan N. (2001), The magical number 4 in short-term-memory: a reconsideration of mental storage capacity. In: Behavioral Brain Science, Bd. 24, S. 87-114.

Cowan N. (1997), Attention and memory. An integrated framework, New York.

Cowan N., Saults J.S., Elliott E.M. (2002), The search for what is fundamental in the development of working memory, Advances in Child Development and Behavior, Bd. 29, S. 1-49.

Cowan N., Towse J.N., Hamilton Z., Saults J.S., Elliott E.M., Lacey J.F., Moreno M.V., Hitch G.J. (2003), Children's working memory processes: A response-time analysis, Journal of Experimental Psychology: General, Bd. 132, S. 113-132.

Daneman M., Carpenter P.A. (1980), Individual differences in working memory and reading, Journal of Verbal Learning and Verbal Behavior, Bd. 19, S. 450-466.

Fanselow G., Kliegl R. \& Schlesewsky M. (1999), Processing difficulty and principles of grammar. In: Kemper S., Kliegl R. (eds.), Constraints on language, Dordrecht, S. 171-201.

Fiebach Ch.J., Schlesewsky M., Friederici A.D. (2001), Syntactic working memory and the establishment of filler-gap dependencies: insights from ERPs and fMRI. In: Journal of Psycholinguistic Research, Bd. 30(3), S. 321-38.

Fiebach Ch.J., Schlesewsky M. \& Friederici A.D. (2002), Separating syntactic memory costs and syntactic integration costs during parsing: the processing of German Wh-questions. In: Journal of Memory and Language, Bd. 47(2), S. 250-272.

Fiebach Ch.J., Vos S.H., Friederici A.D. (2004), Neural correlates of syntactic ambiguity in sentence comprehension for low and high span readers, Journal of Cognitive Neuroscience, Bd. 16(9), S. $1562-75$.

Fodor J.A. (1981), The modularity of mind, Cambridge MA.

Frazier L. (1987), Syntactic Processing: Evidence from Dutch. In: Natural Language \& Linguistic Theory, Bd. 5(4), S. 519-559.

Frazier L. (1988), Grammar and language processing. In: Newmeyer F.J. (ed.), Linguistics : The Cambridge Survey Volume II Linguistic Theory: Extensions and Implications, Cambridge, S. $15-34$.

Frazier L. (1990), Exploring the architecture of language processing system, In: Gerry T.M. Altmann (ed.), Cognitive models of speech processing: Psycholinguistics and computational perspectives, Cambridge, S. 409-433.

Frazier L., Flores D'Arcais G.B. (1989), Filler driven parsing: A study of gap filling in Dutch, Journal of Memory and Language, Bd. 28, S. 331-344.

Friederici A.D. (2009), Pathways to language: fiber tracts in the human brain. In: Trends in Cognitive Sciences, Bd. 13(4), S. 175-181.

Friederici A.D. (2002), Towards a neural basis of auditory sentence processing. In: Trends in Cognitive Sciences, Bd. 6(2), S. 78-84.

Friederici A.D., Bahlmann J., Heim S., Schubotz R.I., Anwander A. (2006), The brain differentiates human and non-human grammars: functional localization and structural connectivity. In: Proceedings of the National Academy of Sciences of the United States of America, Bd. 103(7), S. $2458-63$.

Friederici A.D., Fiebach Ch.J., Schlesewsky M., Bornkessel I.D. \& von Cramon DY. (2006), Processing linguistic complexity and grammaticality in the left frontal cortex. In: Cerebral Cortex, Bd. 16(12), S. 1709-17.

Friederici A.D. \& Kotz S.A. (2003), The brain basis of syntactic processes: functional imaging and lesion studies. In: NeuroImage, Bd. 20, S. 8-S17. 
Friederici A.D., Mecklinger A. (1996), Syntactic parsing as revealed by brain responses: first-pass and second-pass parsing processes. In: Journal of Psycholinguistic Research, Bd. 25(1), S. $157-76$.

Frisch S., Schlesewsky M., Saddy D. \& Alpermann A. (2002), The P600 as an indicator of syntactic ambiguity. In: Cognition, Bd. 85(3), S. B83-B92.

Hashimoto R., Sakai K.L (2002), Specialization in the left prefrontal cortex for sentence comprehension. In: Neuron, Bd. 35, S. 589-597.

Jackendoff R. (2007), A Parallel Architecture perspective on language processing. In: Brain Research, Bd. 1146, S. 2-22.

Just M.A., Carpenter P.A. (1992), A capacity theory of comprehension: Individual differences in working memory. In: Psychological Review, Bd. 98, S. 122-149.

Kluender R., Kutas M. (1993), Bridging the Gap: Evidence from ERPs on the Processing of Unbounded Dependencies. In: Journal of Cognitive Neuroscience, Bd. 5, S. 196-214.

Lin Chien-Jer Ch. \& Bever T.G. (2006), Subject Preference in the Processing of Relative Clauses in Chinese. In: Baumer D., Montero D., Michael S. (eds.), Proceedings of the 25th West Coast Conference on Formal Linguistics, Somerville MA, S. 254-260.

Logie R.H. (1995), Visuo-spatial working memory, Hove.

MacDonald M.C., Christiansen M.H. (2002), Reassessing working memory: comment on Just and Carpenter (1992) and Waters and Caplan (1996). In: Psychological Review, Bd. 109, S. 35-54.

MacDonald M.C., Pearlmutter N.J., Seidenberg M.S. (1994), The lexical nature of syntactic ambiguity resolution. In: Psychological Review, Bd. 101(4), S. 676-703.

Makuuchi M., Bahlmann J., Anwander A., Friederici A. D. (2009), Segregating the core computational faculty of human language from working memory. In: Proceedings of the National Academy of Sciences of the United States of America, Bd. 106(20), S. 8362-8367.

Martin R.C. (1993), Short-term memory and sentence processing: evidence from neuropsychology. In: Memory \& Cognition, Bd. 21(2), S. 176-83.

Meng M., Bader M., Bayer J. (1999), Die Verarbeitung von Subjekt-Objekt-Ambiguitäten im Kontext, Proceedings der 4. Fachtagung der Gesellschaft für Kognitionswissenschaften, St. Augustin: Infix, S. 244-249.

Nicol J.L., Fodor J.D., Swinney D. (1994), Using cross-modal lexical decision tasks to investigate sentence processing. In: Journal of Experimental Psychology: Learning, Memory, and Cognition, Bd. 20, S. 1229-1238.

Nicol J.L. \& Swinney D. (1989), The role of structure in coreference assignment during sentence comprehension. In: Journal of Psycholinguistic Research, Bd. 18, S. 5-19.

Pearlmutter N., MacDonald M.C. (1995), Individual differences and probabilistic constraints in syntactic ambiguity resolution. In: Journal of Memory and Language, Bd. 34, S. 521-542.

Schlesewsky M., Fanselow G., Kliegl R., Krems J. (1999), Preferences for grammatical functions in the processing of locally ambiguous wh-questions in German. In: Hemforth B., Konieczny L. (eds.), German sentence processing, Dordrecht, S. 65-93.

Schriefers H., Friederici A.D., Kühn K. (1995), The Processing of Locally Ambiguous Relative Clauses in German. In: Journal of Memory and Language, Bd. 34, S. 499-520.

Smith E.E., Jonides J. (1998), Neuroimaging analyses of human working memory. In: Proceedings of the National Academy of Sciences of the United States of America, Bd. 95, S. 12061-12068.

Stoltzfus E., Hasher L., Zacks R. (1996), Working Memory and Aging: Current Status of the Inhibitory View. In: Richardson J.T.E., Engle R.W., Hasher L., Logie R., Stoltzfus E., Zacks R.T. (eds.), Working Memory and Human Cognition, Oxford, S. 66-77.

Waters G., Caplan D. (2004), Verbal working memory and on-line syntactic processing: Evidence from self-paced listening. In: The Quarterly Journal of Experimental Psychology Section A: Human Experimental Psychology, Bd. 57(1), S. 129-163. 
Waters G., Caplan D. (1996a), The capacity theory of sentence comprehension. A reply to Just \& Carpenter (1992). In: Psychological Review, Bd. 103, S. 761-772.

Waters G., Caplan D. (1996b), The measurement of verbal working memory capacity and its relation to reading comprehension. In: The Quarterly Journal of Experimental Psychology, Bd. 49A, S. 51-79.

Waters G., Caplan D. \& Hildebrandt N. (1991), On the structure of verbal short-term memory and its functional role in sentence comprehension: evidence from neuropsychology. In: Cognitive Neuropsychology, Bd. 8, S. 81-126.

Zurif E.B., Caramazza A., Myerson R. (1972), Grammatical judgments of agrammatic aphasics. In: Neuropsychologia, Bd. 10, S. 405-417.

\section{Jolanta Sękowska}

\section{SPECIALIZATION OF WORKING MEMORY FOR THE SENTENCE PROCESSING (Summary)}

Constructing dependency relations, in particular subject-verb agreement, in languages in which verbs may occupy the final position in the sentence, requires a combination of newly appearing lexical items with the existing context, such that it extends beyond the limits of the phrase. New words can be integrated only if the part of the sentence that has already been processed remains in a state of activation, being open to receive new elements. This article focuses on the question of the specificity for the domain of linguistic knowledge of the working memory, as the cognitive system in which representations are temporarily stored and manipulated.

Key words: Modularity, sentence processing, sentence comprehension, working memory, domain specificity. 